\title{
A tentative description of the crystallography of amorphous solids
}

\author{
M. Kléman and J. F. Sadoc \\ Laboratoire de Physique des Solides (LA 2), Université Paris-Sud, Bât. 510, 91405 Orsay Cedex, France
}

(Reçu le 26 juillet 1979, accepté le 20 septembre 1979)

\begin{abstract}
Résumé. - On montre que l'on peut obtenir un certain nombre de réseaux aléatoires continus en appliquant de manière particulière des réseaux ordonnés appartenant à des espaces de courbure constante sur l'espace euclidien habituel. Ces applications se caractérisent par l'introduction de deux types de défauts de réseau : défauts de surfaces pour les espaces à courbure constante positive (espaces sphériques), disinclinaisons pour les espaces à courbure constante négative (espaces de Lobatchewski). Cette méthode pourrait apporter des éléments de réponse aux difficultés rencontrées dans la description systématique des amorphes à liaisons métalliques ou covalentes.
\end{abstract}

\begin{abstract}
A number of continuous random lattices can be classified as specific one-to-one mappings of ordered lattices of spaces of constant curvature onto the usual 3-dimensional euclidean space. These mappings put emphasis on two types of lattice defects : surface defects for spaces of constant positive curvature (i.e. spherical spaces), disclinations for spaces of constant negative curvature (Lobatchewskian spaces). This method might provide clues for solving the difficulties encountered in the description of amorphous solids with metallic or covalent bonds.
\end{abstract}

1. Introduction. - There is at the moment a large amount of discussion about the crystallographic properties of amorphous materials (glassy states). We restrict here the consideration to glasses which can be described as resulting from a long range random packing of hard spheres; these materials always present some sort of short range order, for example the occurrence of five numbered rings of bonds [1]. This local arrangement originates in the energetic prevalence of regular tetrahedra of closed packed equal atoms : regular tetrahedra cannot fill space regularly but, at the expense of some slight elastic distortion, assemble into small regular clusters, the simplest type being a set of 5 tetrahedra with a common edge (i.e. a set of 7 atoms). Larger units can be observed, based on the same local arrangement [2]. Other local arrangements exist, for example in covalent solids [3].

In most amorphous metallic solids the average number of atoms in contact with a given atom is slightly smaller than $\bar{z}=12$ [4]. For $\bar{z}=12$ everywhere, one obtains the largest packing density of space filled with a regular set of hard spheres (the fcc lattice), this density being $d=\pi(18)^{-1 / 2}=0.74048 \ldots$ A remarkable aspect of amorphous solids is that the packing density is not very much smaller than the theoretical largest one for the regular lattice. Known analyses of random packings [5] differentiate between loose random packing which reaches a density of $d=0.6$, with $\bar{z}=6$ (more than 6 atoms, however, at a small distance) and (when a vessel containing loosely random packed grains is shaken), close random packing, with $d=0.64$. This is practically the density of packing of amorphous metallic solids. Then a natural question arises; is short range (pentagonal) order enough to generate such a random network ? The answer of most authors is positive. But are there other types of short range order which would also generate randomness, and what are these types? This paper wants to give a (probably) partial answer to these questions.

Two important steps have been made recently in the understanding of the crystallographic (dis)order of amorphous solids, by Sadoc and by Rivier [6]. Let us summarize their findings.

Sadoc starts from the remark that, whereas regular tetrahedra are not space fillers of the 3-dimensional euclidean space $\mathrm{R}_{3}$, they tile regularly the 3-dimensional sphere $S_{3}$ (analytically, $S_{3}$ is the manifold satisfying the equation $\sum_{i=1}^{4} x_{i}^{2}=1$ in $\mathrm{R}_{4}$ ), on which they build a so-called regular polytope. A polytope is nothing else but a $n$-dimensional analog of an ordinary 2-dimensional polyhedron scribed on an ordinary sphere $S_{2}$. (See ref. [7] for classification of polytopes and part 2 of this paper for a brief outline of the subject.) It turns out that the polytope considered by Sadoc contains 600 tetrahedra (5 of them around each edge, as in the 7-atom aggregate considered above), 1200 tri- 
angles, 720 edges, 120 vertices (i.e. 120 atoms). Each vertex has 12 neighboring atoms.

Now it is always possible to map $S_{3}$ (and the polytope vertices scribed on it) on a part of $R_{3}$ (only a part because $S_{3}$ has a finite volume) and obtain an atomic arrangement which has some of the desired properties of an amorphous metal. (We shall sum up these ideas in part. 3.)

Rivier [6] considers the problem from a different point of view; he has established this extremely interesting result : odd-numbered rings of atoms in a continuous random network (this is Zachariasen's terminology for a close random packing) are located along closed lines which cross them but do not cross even-numbered rings. He identifies such (virtual) lines with disclination lines and classifies them as elements of the homotopy group $\pi_{1}\left(E_{3}\right)=Z_{2}$, where $E_{3}$, the euclidean group, is also the manifold of internal states of an amorphous solid (according to his analysis, which at this level is very similar indeed of the analysis done by Toulouse for spin-glasses [8]) and $Z_{2}$ the abelian group of order two. For the use of homotopy groups in physics, see ref. [9].

Both these works clearly proceed from a common belief in the existence in glassy states of some features of order not yet conveniently described. We share this belief and propose an answer which generalizes both. Our analysis will ultimately lead to a classification of continuous random networks, " a goal which has occupied mathematicians from the seventeenth century onwards » (Bernal), without being reached, since they are interested in filling space with polyhedra. The key concepts will be crystallographic in nature, not in euclidean space, but either in spherical space (constant positive curvature) as pointed out above, or in the simply-connected hyperbolic space (constant negative curvature) as introduced below; defects will play an essential physical role in this crystallography, in a much more dramatic way than usual, but they do not appear in the same way in spaces of positive and negative curvatures.

These concepts will be discussed in part 3 for spherical spaces and in parts 4, 5 and 6 for spaces of negative curvature.

2. Regular lattices in curved spaces. - 3-dimensional curved spaces with constant curvatures can be tiled with cells consisting in regular polyhedra, achieving in this way a so-called regular honeycomb. Here the word regular means that all the faces of each polyhedron are equal and that all the edges of each face are equal. The standard notation which covers the various cases is the Schläfli symbol $\{p, q, r\}(p, q, r$ integers) where $p$ is the symmetry order of a face ( $p=3$ for an equilateral triangle, $p=4$ for a square), $q$ the number of faces around a vertex in a cell $(p=3$, $q=3$ define a regular tetrahedron, $\{4,3\}$ a cube); more generally $\{p, q\}$ defines a cell, i.e. a regular polyhedron, and $\{p, q, r\}$ an assembly of $\{p, q\}$ cells, $r$ of which around a common edge.

There are five regular polyhedra

$$
\begin{array}{ccccc}
\{3,3\} & \{4,3\} & \{3,4\} & \{3,5\} & \{5,3\} \\
\text { tetrahedron } & \text { cube } & \text { octahedron } & \text { icosahedron } & \text { dodecahedron }
\end{array}
$$

and only five because, since the characteristic angle of a regular $p$-gon is $\left(1-\frac{2}{p}\right) \pi$, since $q$ of them have to fit around a common vertex, and since the angle of a spherical $\{p\}$ is greater than $\left(1-\frac{2}{p}\right) \pi$, this yields

$$
\left(1-\frac{2}{p}\right) \pi<\frac{2 \pi}{q}
$$

or

$$
(p-2)(q-2)<4 \text {. }
$$

All other sets $\{p, q\}$ which do not satisfy (1), either describe a regular honeycomb of the euclidean plane :

$$
(p-2)(q-2)=4 \rightarrow \begin{array}{ccc}
\{4,4\} & \{3,6\} & \{6,3\} \\
\text { sets of squares, } & \text { of triangles, } & \text { of hexagons }
\end{array}
$$

or a regular honeycomb of the hyperbolic (Lobatchewskyan) plane :

$$
(p-2)(q-2)>4 \rightarrow \text { these sets }\{p, q\} \text { are infinite in number } .
$$

In other words, the Schläfli symbol $\{p, q\}$ describes as well polyhedra and honeycombs in two dimensional curved spaces.

Now let us join $r$ regular polyhedra around a common edge. Schläfli's trigonometrical condition reads as follows : the quantity

$$
\sin \frac{\pi}{p} \sin \frac{\pi}{r}-\cos \frac{\pi}{q}
$$


is positive for a spherical space, negative for an hyperbolic space, null for an euclidean space. There are :

- six spherical polytopes

$$
\{3,3,3\} \quad\{3,3,4\} \quad\{4,3,3\} \quad\{3,4,3\} \quad\{3,3,5\} \quad\{5,3,3\}
$$

- one euclidean honeycomb (only), made of cubes

$$
\{4,3,4\}
$$

- eight hyperbolic honeycombs

$\{6,3,3\} \quad\{5,3,4\} \quad\{6,3,4\} \quad\{4,4,3\} \quad\{4,3,5\} \quad\{3,5,3\} \quad\{5,3,5\} \quad\{6,3,5\}$.

Apart from regular honeycombs (or polytopes), it will be interesting to consider ordinary lattices and quasi-regular polytopes. They can be classified by their group of symmetry. We have space groups in spherical or hyperbolic space, as we have Schönflies groups in euclidean space. The space groups in spherical space have finite order (there are no translations); their order is infinite in hyperbolic space.

It is not possible to give here even a short description of space groups in curved spaces. For details see ref. [10].

3. Amorphous tetrahedral packing and the polytope $\{3,3,5\}$. - Let us consider tetrahedral packing, as it is an important physical case, and consider possible polytopes whose mappings on euclidean space would generate amorphous materials of this type. We have three possibilities $\{3,3,3\},\{3,3,4\}$ and $\{3,3,5\}$. But only the last one is attractive, because each vertex has a reasonable amount of neighbors $(\bar{z}=12)$, while $z=4$ for $\{3,3,3\}$ and $z=6$ for $\{3,3,4\}$. Also the packing density is larger in $\{3,3,5\}$ than in any of the other polytopes [5].

The polytope $\{3,3,5\}$ contains 600 tetrahedra and 120 vertices. Each vertex, being surrounded by 12 neighbors, is the center of an icosahedron. If each vertex is replaced by an atom, one obtains an excellent approximation (although in a curved space) of the amorphous dense-packed structure described in refs. [1] and [2].

Now it is always possible, in fact in many ways as we shall discuss in the next paragraph, to map the sphere $S_{3}$ and the $\{3,3,5\}$ polytope scribed on it, on a part (since $S_{3}$ is finite) of the euclidean space $R_{3}$. Such a mapping will necessarily introduce non-homogeneous distortions in the lengths. These distortions are sources of elastic stresses in the euclidean physical space. But they are not too large, because $S_{3}$ is small and involves only 120 atoms. As for the surface $\Sigma$ limiting in $R_{3}$ the mapping we have performed, it is the image in this mapping of the lips of a two dimensional cut in $S_{3}$. This cut is the exact analogue (in 3 dimensions) of the one dimensional cut we would have to perform on a sphere $S_{2}$ in order to map it on a plane. Peeling an orange is a practical example of such a cut, from which case we see by extrapolation to 3 dimensions that we are left with numerous possibilities in the choice of $\Sigma$.

In summary, the mapping of $S_{3}$ on $R_{3}$ induces

- elastic distortions which keep the local icosahedral order unchanged,

- a closed wall, the image of $\Sigma$, surrounding the cluster of 120 atoms, with a higher randomness of the atoms and a local discontinuity of order.

These two kinds of effects are correlated. It is clear that the larger the closed wall area, the smaller the elastic distortions, and the larger the packing density. The total packing density of an amorphous medium is obtained as an average on a set of images of $S_{3}$ in contact along walls.

It is possible to achieve a contact between two images in order to make a perfect fitting at the wall, without change in local order, but not to suppress the higher disorder at triple junctions $\left({ }^{1}\right)$. With such a model, one of us (J.F.S.) has calculated the packing fraction as a function of a single parameter with which it is possible to define the mapping, viz. the elastic distortion, and has found that, whereas the bulk contribution to this packing fraction decreases with increasing elastic distortion, the wall contribution increases. A computed model leads to an optimum value of $d: 0.63$, in good agreement with the experimental value in hard sphere packing. J.F.S. will publish this model in a future paper.

4. On mappings between spaces of different curvatures. - This short qualitative discussion of a possible model for close random tetrahedral packing, based on the use of $\{3,3,5\}$, has shown how 2 dimensional defects are introduced naturally in this case, essentially because $S_{3}$ is a finite manifold. But, as shown by M. Kléman, in the most general case of mapping between different manifolds, the defects which are introduced naturally are linear defects, more precisely : disclinations. This can be demonstrated as follows.

Let us consider a three-dimensional Riemannian manifold $M\left(S_{3}\right.$ is such an object) and map it on $R_{3}$.

( $\left.{ }^{1}\right)$ Therefore one can join any two points in the amorphous medium by a path which meets the same local order all along. 
Many ways exist of doing this, some of them being likely to have more physical meaning than others. For example it is always possible to map $\mathbf{M}$ conformally (i.e. conserving angles) on $R_{3}$, in a one-to-one mapping; but, when applied to $S_{3}$, such a mapping changes the lengths quite drastically. A geodesic mapping (transforming geodesic lines of $\mathbf{M}$ to straight lines in $\mathbf{R}_{\mathbf{3}}$ ), although not possible in the general case, exists between $S_{3}$ and $R_{3}$. However it distorts lengths (in fact more than the conformal mapping). An isometric mapping (conserving distances between atoms in $\mathbf{M}$ ) would be the most exciting thing to do, but unfortunately is not a one-to-one mapping. It is however this mapping that we want to use, so that we have first to recognize the nature of this multivaluedness. The theorem of Riemannian geometry which is at the heart of our analysis reads as follows :

Theorem $[11,12]:$ the parallel transport of vectors from one point of a Riemannian manifold to another point along a fixed path $\mathrm{C}$ in a linear isometric operation.

We shall not define with precision the various concepts which we use here : intuitively, this theorem means that it is possible to roll (without gliding) a Riemannian manifold on an euclidean manifold along a fixed path (think of a conventional sphere $S_{2}$, of any line on $\mathrm{S}_{2}$, and of a plane), such that $a$ ) the distances along the path are conserved, $b$ ) the angles between vectors attached to the path and the path itself are conserved. In particular, if $\mathrm{C}$ is a geodesic of $M, M$ rolls along a straight line of $R_{3}$, and vectors which are parallel (in the mapping, i.e. in the euclidean sense) are mappings of parallel vectors (on $\mathbf{M}$, i.e. in the Riemannian sense).

Of course, if all the possible paths of $\mathbf{M}$ could be rolled down on the same Euclidean manifold in a one-to-one mapping, we would have complete isometry, and we would say that $M$ is developable on $R_{3}$. But this is not the general case.

Now consider a fixed infinitesimal closed path $\mathrm{C}$ on $\mathrm{M}$; this path being small is defined at a particular point $\mathbf{P}$ of $\mathbf{M}$ (for example $\mathbf{P}$ is a particular point on $\mathrm{C}$ ) and is perpendicular to a given direction $v$, of director cosines $\alpha_{i}$ in some local frame of coordinates. When rolling $M$ on $R_{3}$ along $C$, from $P$ to $P$, one obtains in general an open path, and any frame of reference attached to $\mathbf{P}$ and followed by parallel transport is rotated at the end of the path by an angle

$$
\Omega=K \sigma
$$

$\sigma$ is the small area enclosed by the loop on $\mathbf{M} ; K$ is a finite quantity called the sectional curvature; the displacement of $P$ reads

$$
D=\Omega \lambda
$$

where $\lambda$ is the diameter of $\mathrm{C}$.

Clearly, the displacement $D$ is second order, the rotation first order. What we have created in $R_{3}$ is a disclination of strength $\Omega$, centered on some point interior to $\mathrm{C}$, because this operation, defined here at a point, can be extended (with the same strength $\Omega$ ), to a closed line (the standard interpretation of Bianchi's identities is that disclination lines are loops). The mapping $\mathbf{M} \rightarrow \mathbf{R}_{\mathbf{3}}$ is isometric along $\mathrm{C}$, but is not closed. We close it by inserting some matter in the void (if $K$ is positive), or removing some extra matter (if $K$ is negative). Of course such extra matter, which is unambiguously defined in $\mathbf{M}$, needs to be mapped in its own way on $R_{3}$, and this operation will certainly perturb the distances between atoms. But, as long as $\lambda$ is small, this is a small perturbation.

Finally, the entire mapping $\mathbf{M} \rightarrow \mathrm{R}_{3}$ can be achieved by creating in $\mathbf{M}$ three families of disclination densities, in 3 orthogonal directions, defined by rotation vectors per unit area

$$
\omega_{i}=K_{j i} \alpha_{i}
$$

$K_{i j}$ is the tensor of curvature, $K=\omega_{i} \alpha_{i}$ the already defined sectional curvature. This very special kind of mapping (by disclinations) can be approached by finite disclinations, whose strengths would correspond to the elements of rotational symmetry of the regular lattice of atoms on the Riemannian manifold $\mathbf{M}$. It is therefore not unique.

5. The choice of $\mathbf{M}$. - In the case of small aggregates, $M=S_{3}$, adorned with a $\{3,3,5\}$ honeycomb, appears as a good candidate, but the mapping we used is not in the disclination mode. Note moreover that, if we performed such a mapping by disclinations, it would lead to an euclidean medium with $z>12$, because the sectional curvature $K$ is positive for any direction (and is in fact constant, $K=+1$ is the radius of the sphere and $S_{3}$ has constant curvature). This is not realistic. Hence other honeycombs, for example $\{3,3,3\}$ or $\{3,3,4\}$, or eventually non-regular lattices with coordination number $z<12$, should be preferred for the disclination mode. If so, the overall obtained material would have to be defined by linear and surface defects, altogether. This would be a very complex picture of an amorphous material. Let us investigate other possibilities.

There is of course a great physical interest in choosing for $\mathbf{M}$ a space of constant (sectional) curvature. Such a space shares with the euclidean space (of zero curvature) many properties; a) There is a continuous group $\mathrm{G}$ of isometries depending on 6 parameters under which such a space is invariant. It is the euclidean group $\mathbf{E}_{\mathbf{3}}$ for $\mathbf{R}_{\mathbf{3}}$. b) This implies properties of isotropy and homogeneity : any object drawn on a space of constant curvature can be moved without deformation in any direction, and turned to any orientation; $c$ ) Such a space can bear a regular lattice of points (with a pattern eventually) which is invariant under a subgroup $\mathrm{H}$ of $\mathrm{G}$. For the euclidean space there are 230 such space groups, or Schönflies group ; $d$ ) One can define, in such a lattice, defects 
in exactly the same way as in $R_{3}$, i.e. by the homotopy groups [13] $\Pi n(\mathrm{G} / \mathrm{H})$ but also, because we have a group $G$ of rigid displacements, by the Volterra process.

Hence mapping a space of constant curvature in $\mathbf{R}_{\mathbf{3}}$ as isometrically as possible consists in introducing a number of disclination lines in $\mathbf{M}$, whose individual strength is related to the relevant Volterra process, and whose total strength counterbalances, as in eq. (2), the curvature of the planar section to which they are perpendicular. Then the mapping $\mathbf{M} \rightarrow \mathbf{R}_{3}$ can be made one-to-one and practically isometric.

Now, since we have already discussed the $3 d$ spherical space, we are left with the only other possibility, viz. the simply connected $3 d$ hyperbolic space of constant negative curvature $K=-1$. This space (let us call it $\mathrm{H}_{3}$ ) is diffeomorphic to $\mathrm{R}_{3}$; hence it is possible to devise a one-to-one mapping between them. Finally, since $K$ is negative, any mapping in the disclination mode decreases the coordination number.

If this model of amorphous materials is correct, their ground state is defined by a density of disclination lines which approaches (in the sense of eq. (2)) the right curvature contribution necessary to fill the gap between $K=-1$ and $K=0$. The choice of these disclinations is submitted to the condition that the rest of the mapping (the non-isometric part which brings elastic distortions to the medium) optimizes the energy.

6. Disclinations. - It therefore appears that Rivier's idea of looking for the existence of well individualized closed lines is physically correctly rooted. But are they disclinations, and more of that, are they correctly defined as being representatives of $\Pi_{1}\left(\mathrm{E}_{3}\right)=\mathrm{Z}_{2}$ ?

Let us first assume that this is true. Hence, by some process of recovery, they could anneal two by two. In fact, since they are unpolar defects (because of $Z_{2}$, any line is equal to its antiline), as long as there are more than two defects they anneal in this way, and finally not more than one defect would subsist, which eventually would disappear by self annealing. Therefore, if one follows Rivier's analysis to its end, there would exist an ordered amorphous medium, without defects, whose symmetry group is $\mathbf{H}=1$, and which is globally invariant under any operation of $E_{3}$, the euclidean group. It is evident that such a medium does not exist.

Therefore, if we still accept Rivier's result i.e. that the lines he has discovered are disclinations there must be some strong impossibility to the annealing of defects. This is so because the defects are classified as elements of the homotopy groups of $\mathrm{G}\left(\mathrm{H}_{3}\right) / \mathrm{H}$. This difficulty of annealing is not of topological origin : since $\mathbf{H}_{3}$ and $\mathbf{R}_{3}$ are diffeomorphic, it is perfectly feasible to consider a one-to-one mapping of the process of annealing. But, after such an annealing, $\mathbf{R}_{\mathbf{3}}$ would not be tesselated by an ordered lattice, since there is no one-to-one isometry between $R_{3}$ and $\mathrm{H}_{3}$. The energy of this undislocated phase would be very high : the obstruction to the annealing of defects is of thermodynamic origin, via an order parameter which is non-euclidean. In the language of groups of symmetry, this also means that the spacegroups of $\mathrm{H}_{3}$ are not isomorphic to any of the Schönflies groups.

Let us notice that the same arguments apply to frustration in spin-glasses; these defects have been classified by Toulouse [8] as elements of $\Pi_{1}(\mathrm{SO}(3))$. But this cannot be true, since otherwise it would be possible to obtain, by motion of frustration lines and their annealing, a ground state of a spin-glass without frustration. Frustration must probably be classified by homotopy elements of the quotient spaces of some non-euclidean space.

After such an analysis, it is not too audacious to establish a link between non-ordered media and phases whose symmetry group is not a subgroup of $E_{3}=G\left(R_{3}\right)$. This conception should open new fields of research in the domain of phase transitions.

Finally let us come back to a description of amorphous materials in terms of defects. Disclinations, as we have seen, are thermodynamically necessary (hence Rivier is right in giving this name to the closed lines he has discovered); but other defects might exist in the $\mathrm{H}_{3}$-hypothetical state, which would appear as true defects by the one-to-one mapping : dislocations (which would be here more safely called by a specific name : disvections, since translations (transvections) are not commutative in $\mathrm{H}_{3}$ ), wedge and twist disclinations (these latter relaxed by disvections), walls, configurations, etc... Note that wedge disclinations are sufficient to achieve the mapping by disclinations.

7. Conclusion, further comments. - i) We have stressed that $\{3,3,5\}$ is a good candidate for tetrahedral packing. Since $\{3,3,5\}$ is a honeycomb on $\mathrm{S}_{3}$, an amorphous solid would therefore be a nonhomogeneous medium, made of small clusters. However the walls separating these clusters might be energetically unstable towards a transformation in a lattice of lines. This remains to be tested, experimentally and theoretically.

$\{3,5,3\}$ is an hyperbolic honeycomb : three icosahedra around each edge, $z=20$. By putting an atom at the center of these icosahedra, and introducing a number of disclinations, this can be a good candidate also for tetrahedral packing. All nodes of this centered $\{3,5,3\}$ are not equivalent. Here too a detailed study remains to be done. This glass would be homogeneous.

One might also expect that the various space groups of $\mathrm{H}_{3}$ might furnish models for covalent glasses.

ii) The most striking aspect in our analysis is the physical impossibility for disclinations to anneal together. This implies that atoms are only slightly 
diffusive, and this might be related to the concept of localization introduced by Anderson [14].

iii) Our analysis in terms of disclinations (for $\mathrm{H}_{3}$ ) is just the opposite of the usual analysis, in which, given an ordered medium (of $R_{3}$ ) containing disclinations, a curved space is attached to this disclinated medium [15]. But this standard analysis is just a convenient way of describing disclinations in a flat space. On the contrary the physics of our curved space is precisely contained in its curvature (its sign), and in its space-group.

iv) An amusing consequence, at a cosmological level, of our analysis, would be as follows. Consider an homogeneous isotropic system of particles in relative uniform motion in an euclidean space according to Hubble's law, and assume that the law of composition of velocities is the law of Einstein in special relativity. It can be shown [10] that such a problem can be reduced to the purely geometrical problem of constructing an homogeneous honeycomb in a space of constant negative curvature $\mathrm{H}_{3}$. Therefore if the (real) space is euclidean, it is a mapping of $\mathrm{H}_{3}$ : it must probably contain a density of disclinations of the lattice of galactic clusters. If it is not an euclidean space, this density would be different, and its study would reveal space curvature. But astrophysicists might object to such a conclusion !

\section{References}

[1] Sadoc, J. F., Dixmier, J. and Guinier, A., J. Non Cryst. Solids 12 (1973) 46.

[2] Farges, J., Thesis (1978), Orsay.

Farges, J., DE Féraudy, M. F., RaOUlt, B. and Torchet, G., J. Physique 36 (1975) 62.

[3] PolK, D. E., J. Non Cryst. Solids 5 (1971) 365.

[4] Chaudhari, P. and Turnbull, D., Science 199 (1978) 11.

[5] Fejes Tóth, L., Regular figures (Pergamon Press) 1964.

[6] Rivier, N., Disclination lines in glasses, submitted to Philos. Mag.

[7] Hilbert, D. and Cohn-Vossen, S., Geometry and the Imagination (Chelsea Pub. Cy New York) 1952.

[8] Toulouse, G., Phys. Reports 49 (1979).
[9] Toulouse, G. and Kléman, M., J. Physique Lett. 37 (1976) L-149.

[10] Coxeter, H. S. M. and Whitrow, G. J., Proc. R. Soc. A 201 (1950) 417

[11] ARNol'D, V., Méthodes mathématiques de la mécanique classique (Editions de Moscou) 1974.

[12] Cartan, E., Leçons sur la Géométrie des Espaces de Riemann (Gauthier-Villars, Paris) 1963.

[13] Kléman, M., Michel, L. and Toulouse, G., J. Physique Lett. 38 (1977) L-195.

[14] Anderson, P. W., Phys. Rev. 109 (1958) 1492.

[15] Bilby, B. A., Prog. Sol. Mech. 1 (1960) 329. 